\title{
Probing sterile neutrinos and axion-like particles from the Galactic halo with eROSITA
}

\author{
Ariane Dekker, ${ }^{a, b, *}$ Ebo Peerbooms, ${ }^{b}$ Fabian Zimmer, ${ }^{a}$ Kenny C. Y. $\mathbf{N g}^{c}$ and \\ Shin'ichiro Ando ${ }^{a, b, d}$ \\ ${ }^{a}$ GRAPPA Institute, University of Amsterdam, 1098 XH Amsterdam, The Netherlands \\ ${ }^{b}$ Institute for Theoretical Physics, University of Amsterdam, 1098 XH Amsterdam, The Netherlands \\ ${ }^{c}$ Department of Physics, The Chinese University of Hong Kong, Shatin, Hong Kong China \\ ${ }^{d}$ Kavli Institute for the Physics and Mathematics of the Universe (Kavli IPMU, WPI) University of Tokyo, \\ Kashiwa, Chiba 277-8583, Japan
}

E-mail: a.h.dekker@uva.nl

The nature of dark matter remains an open question and could be in the form of warm dark matter. Sterile neutrinos and axion-like particles are well motivated warm dark candidates, and can decay into photons, which are consequently detectable by X-ray telescopes with keV dark matter mass. Both particles could explain the observed unidentified $3.5 \mathrm{keV}$ line and, interestingly, XENON1T observed an excess at a few $\mathrm{keV}$ that can originate from axion-like particles. We study the diffuse emission coming from the Galactic halo, and test the sensitivity of all-sky X-ray survey eROSITA to identify a sterile neutrino or axion-like particle. By Monte Carlo method, we set bounds on the mixing angle of the sterile neutrinos and coupling strength of the axion-like particles. With eROSITA, we will be able to set stringent constraints, and in particular, we will be able to firmly probe the best-fit of the unidentified $3.5 \mathrm{keV}$ line, where we reach an order of magnitude better sensitivity. Moreover, eROSITA is able to confirm an axion-like particle origin of the XENON1T excess for an excess greater than $3 \mathrm{keV}$.

$37^{\text {th }}$ International Cosmic Ray Conference (ICRC 2021)

July 12th - 23rd, 2021

Online - Berlin, Germany

\footnotetext{
*Presenter
} 


\section{Introduction}

Sterile neutrinos are well motivated dark matter candidates, and can solve some issues in the standard model. They have right-handed chirality, in contrary to the standard model neutrinos which have left-handed chirality. This feature gives a natural explanation for the standard model neutrinos to have mass, and moreover, it can solve the matter-antimatter asymmetry of the universe. Through mixing with active neutrinos, sterile neutrinos can decay into a standard model neutrino and a photon with energy $E_{\gamma}=m_{v_{s}} / 2$, with $m_{v_{s}}$ the sterile neutrino mass, which photon is observable as a monochromatic line signal by X-ray telescopes. Axion-like particles (ALP) are pseudo-NambuGoldstone bosons that can emerge when a continuous global symmetry is spontaneously broken. ALPs can couple to standard model particles through coupling, $g$, and decay into two photons, producing likewise a monochromatic line signal in X-rays.

An emission line at $\sim 3.5 \mathrm{keV}$ was detected through a stacked X-ray spectrum analysis of 73 galaxy clusters [1], suggesting experimental evidence for a dark matter decay signal which could be interpreted as decaying sterile neutrino with mass $m_{v_{s}} \sim 7 \mathrm{keV}$ and mixing angle $\sin ^{2}(2 \theta) \approx$ $\left.(0.2-2) \times 10^{-10}\right)$ [2]. Follow-up studies both confirmed the emission line [3-5], as well as provide strong constraints on decaying dark matter due to non-detections [6-9].

As another interesting possibility, decaying ALP could explain the observed excess of recoil events over known backgrounds at the XENON1T experiment, which is most prominent at 2$3 \mathrm{keV}$. Since the ALP coupling to standard model particles is already tightly constrained by X-ray observations [10] in order to explain this excess, a model is required in which the photon coupling is suppressed. We follow an anomaly-free symmetry model, in which the ALP is coupled to leptons without any anomalous coupling to photons, and where photons are only induced through threshold corrections.

In this work, we estimate the sensitivity of eROSITA's all-sky X-ray survey to observe decaying sterile neutrino and ALP signals. eROSITA will observe the full sky during four years with an average exposure of $2.5 \mathrm{ks}$, and moreover, due to its excellent angular and energy resolution, it is a valuable probe for dark matter decay with narrow X-ray line emissions. In order to obtain the largest dark matter induced X-ray flux, we study the diffuse emission coming from the Galactic halo. By simulating all-sky X-ray count maps, we make a sensitivity projection for eROSITA to a sterile neutrino and ALP signal under a background-only hypothesis. We find that eROSITA allows us to probe for a much large parameter space for both sterile neutrino and ALP dark matter.

\section{X-ray sky map}

\subsection{Sterile neutrino signal}

$\mathrm{X}$-ray photons from sterile neutrino decay inside the Galactic halo produce a flux which depends on the sterile neutrino decay rate $\Gamma_{v_{s}}$, sterile neutrino mass $m_{v_{s}}$, energy spectrum $d N_{\text {decay }} / d E$ per decay, and the $\mathrm{D}$-factor, $D$. The $\mathrm{X}$-ray photon flux from a region $d \Omega$ is given as follows,

$$
\frac{d \Phi}{d E}=\frac{\Gamma_{v_{s}}}{4 \pi m_{v_{s}}} \frac{d N_{\text {decay }}}{d E} D
$$

for which we adopt a delta function for the energy spectrum per decay. The D-factor describes the dark matter density profile of the Milky Way halo, integrated over the line of sight, and we consider 
both the spherically-symmetric Navarro-Frenk-White (NFW) [11] profile and a cored profile [12]. The decay rate depends on the sterile neutrino mass, $m_{v_{s}}$, and mixing angle $\theta$, and is given by the following decay rate $[13,14]$,

$$
\Gamma_{v_{s}}\left(m_{v_{s}}, \theta\right)=1.38 \times 10^{-29} \mathrm{~s}^{-1}\left[\frac{\sin ^{2}(2 \theta)}{10^{-7}}\right]\left(\frac{m_{v_{s}}}{1 \mathrm{keV}}\right)^{5} .
$$

Moreover, we include the extragalactic component for completeness, however, we find that the extragalactic flux is more than an order of magnitude smaller than the Galactic flux within the small energy bins that we adopt in this paper.

\subsection{Axion-like particle signal}

ALP can couple to photons and produce 2 photons with the following decay rate [15]

$$
\Gamma_{a \rightarrow \gamma \gamma} \simeq 5 \times 10^{-29}\left(\frac{m_{a}}{7 \mathrm{keV}}\right)^{3}\left(\frac{f_{a}}{5 \times 10^{14} \mathrm{GeV}}\right)^{-2} \mathrm{~s}^{-1},
$$

where $m_{a}$ is the ALP mass and $f_{a}$ is the decay constant, where we adopt the following relation with the photon coupling $g_{a \gamma \gamma}$,

$$
f_{a} \equiv \frac{\alpha C_{a \gamma \gamma}}{2 \pi g_{a \gamma \gamma}}
$$

with $C_{a \gamma \gamma}=8 / 3-1.92 \approx 0.75$ [10].

In order to explain the XENON1T excess by ALP, we adopt an anomaly-free symmetry model, where photons are induced through threshold corrections. The decay rate is given by [16]

$$
\Gamma_{a \rightarrow \gamma \gamma} \simeq 3.5 \times 10^{-57} \mathrm{GeV}\left(\frac{m_{a}}{2 \mathrm{keV}}\right)^{7}\left(\frac{g_{a e}}{5 \cdot 10^{-14}}\right)^{2},
$$

with $g_{a e}$ the coupling between the ALP and electron. The energy spectrum of the ALP decay is also described by a delta function, which allows for a direct comparison with the sterile neutrino flux and we use the obtained X-ray bounds on the mixing angle and convert to the coupling strength $g_{a \gamma \gamma}$ or $g_{a e}$.

\subsection{Backgrounds}

The cosmic X-ray background contributes to the diffuse emission and we consider a power-law with photon index $\Gamma=1.42 \pm 0.03$ and with a normalization at $1 \mathrm{keV}$ of $8.44 \pm 0.24$ photon $\mathrm{cm}^{-2} \mathrm{~s}^{-1} \mathrm{keV}^{-1} \mathrm{sr}^{-1}$ [17]. Moreover, eROSITA's detector background consist of high energy particles and show a flat spectral energy distribution with a normalization of $3.5 \times 10^{-4}$ counts $\mathrm{keV}^{-1} \mathrm{~s}^{-1} \operatorname{arcmin}^{-2}$ [18]. Besides these two isotropic background components, we consider the X-ray bubbles, which flux is subdominant with respect to eROSITA's detector background below $\sim 2.3 \mathrm{keV}$. The X-ray bubbles are observed by eROSITA with an average count rate in the $0.6-1 \mathrm{keV}$ energy band of 0.0038 photons s${ }^{-1} \operatorname{arcmin}^{-2}$ and 0.0026 photons s$^{-1} \operatorname{arcmin}^{-2}$ in the northern and southern bubbles respectively [19], and we consider an uniform template of the Fermi bubbles for its morphology, downloaded from https://fermi.gsfc.nasa.gov/ssc/data/access/. Finally, in order to exclude the extended emission from the Galactic plane, we remove all pixels with $|b|<20^{\circ}$. 


\section{Analysis}

We consider in total 13 energy bins around $m / 2$ with bin size $\sigma_{E}$, where $\sigma_{E}$ is related to the full width at half maximum through FWHM $=2 / \sqrt{2 \ln 2} \sigma_{\mathrm{E}}$ with FWHM $=138 \mathrm{eV}$ for eROSITA [20], for each sterile neutrino and ALP mass, and range their mass between 2 and $20 \mathrm{keV}$. We generate 500 mock data sets, $n_{i}$, assuming background only through Monte Carlo simulations for each dark matter mass point, where $i$ runs over the energy bins as well as the spatial pixels. The likelihood to obtain $n_{i}$ as a function of the decay rate for a specific mass is given by the likelihood functions:

$$
\mathcal{L}(\Gamma)=\prod_{i} P\left[n_{i} \mid \mu_{i}(\Gamma)\right]=\prod_{i} \frac{\mu_{i}(\Gamma)^{n_{i}} e^{-\mu_{i}(\Gamma)}}{n_{i} !},
$$

where $\mu_{i}(\Gamma)$ are the expected counts in each bin under the signal hypothesis with decaying dark matter and background component. We determine the best-fit model under a maximum likelihood estimation with the test statistic (TS), and obtain upper limits on the decay rates at $95 \%$ confidence level (CL), which corresponds to a test statistic of TS $=2.71$.

\section{Results}

The sensitivity of eROSITA on the mixing angle as a function of the sterile neutrino mass is presented in figure 1. The two green bands show the $68 \%$ and $95 \%$ containment regions from the Monte Carlo runs, whereas the green solid line represents the median with the NFW profile. The orange dashed dotted line illustrates the median of the Monte Carlo runs with a cored profile, and we find little dependence on the density profiles. The lower grey area is excluded by theory due to dark matter underproduction [21,22], while the upper grey area is excluded by current X-ray observations [8, 23-29]. Moreover, the best-fit of the unidentified $3.5 \mathrm{keV}$ line by Ref. [1] is indicated as a black star, with mass $m_{v_{s}}=7.1 \mathrm{keV}$ and mixing angle $\sin ^{2}(2 \theta)=7 \times 10^{-11}$. We find that with an exposure time of $T=2.5 \mathrm{ks}$, eROSITA will be sensitive to the $3.5 \mathrm{keV}$ line and can even constrain the mixing angles up to nearly two orders of magnitude below the best-fit at $m_{v_{s}}=7.1 \mathrm{keV}$.

The sensitivity of eROSITA to the photon coupling is shown in Fig. 2a, where the best-fit for the $3.5 \mathrm{keV}$ line is indicated by the black star. Even though recent work found no evidence for an unassociated X-ray line [8], in which the current X-ray limits are shown as the grey shaded area (see [8, 23-29]), eROSITA will be able to probe a region of the parameter space not yet excluded by current X-ray limits.

Moreover, we test if eROSITA will be able to probe the parameter space relevant for the observed XENON1T excess, which has been observed to be most prominent at ALP mass of $m_{a}=2-3 \mathrm{keV}$ and electron coupling $g_{a e} \sim 10^{-13}$. An anomaly-free ALP model is adopted, for which the expected sensitivity is presented in Fig. 2b. The excluded region based on X-ray observations are in grey, and indeed, we probe a parameter space not yet constrained [8, 23-29]. The expected sensitivity of Athena taken from Ref. [30] show comparable sensitivity, as shown as the red dashed dotted line. The black solid line shows the XENON1T limit at 90\% CL [31], and the dark grey box highlights roughly the best-fit parameter space from Ref. [32]. Future eROSITA data may not reach the best-fit for the XENON1T excess, however, if the best-fit alters towards higher 


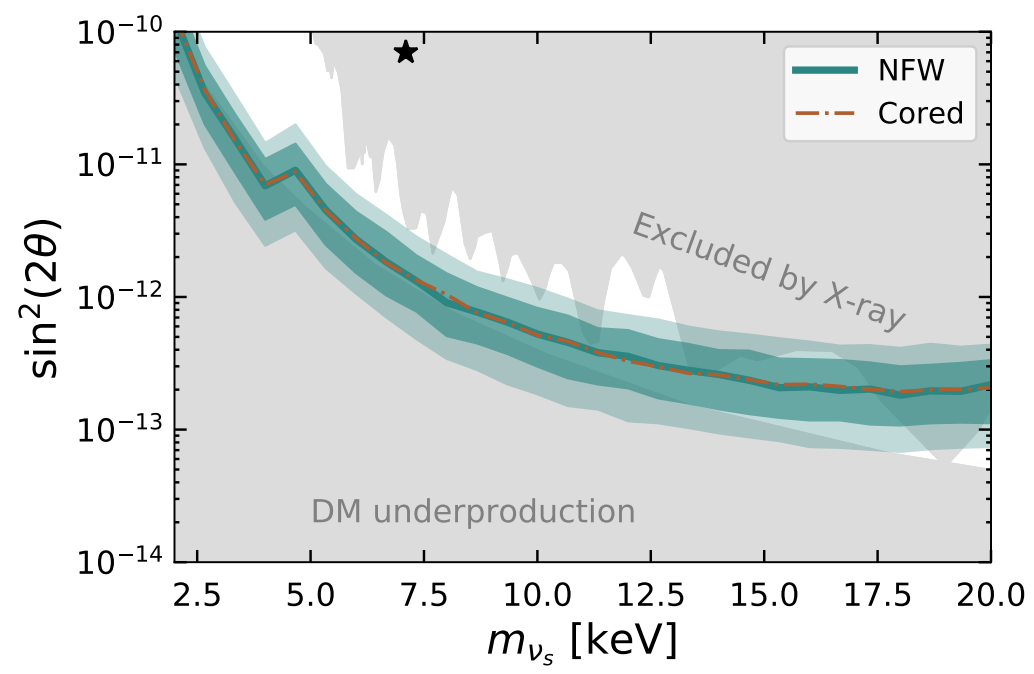

Figure 1: Sensitivity to the mixing angle as a function of the sterile neutrino mass for the cored (dashed orange) and NFW (green) profiles, showing the 68\% and 95\% containment regions of the sensitivities and the median (solid line) from the Monte Carlo runs with an NFW profile.

ALP mass, still inside the XENON1T excess region of interest with energies between 1-7 keV, an ALP origin could be confirmed. Interestingly, comparison with the expected sensitivity of Athena taken from Ref. [30] shows similar sensitivity. The yellow shaded area illustrates the preferred region for the white dwarf cooling anomaly, whereas the yellow dotted line illustrates the preferred values for the red giant branch in globular clusters [32-34]. These stellar cooling anomalies are observed excesses in cooling of stellar objects, which could be explained by ALP, and their preferred regions are close to the XENON1T excess.

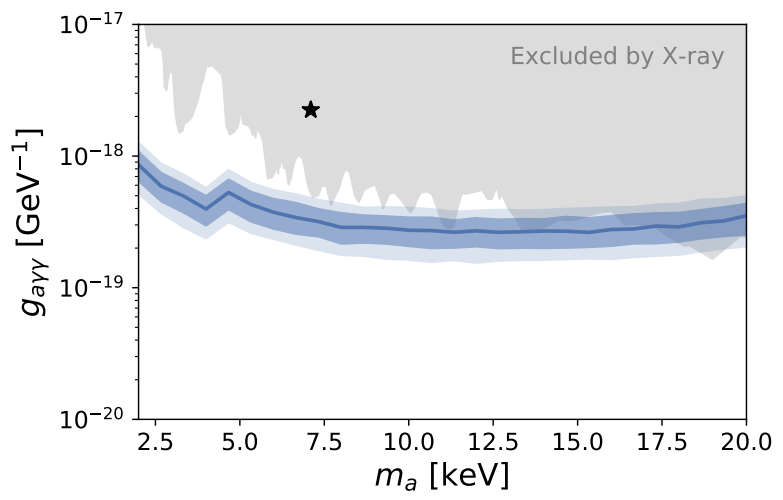

(a) ALP-photon coupling

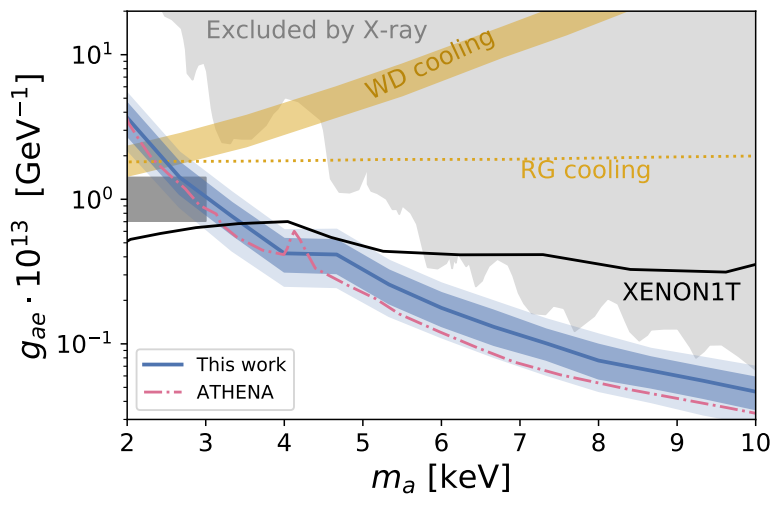

(b) ALP-electron coupling

Figure 2: Current and future limits on the ALP-photon coupling (a) and ALP-electron coupling (b). The blue bands show the 1 and $2 \sigma$ sensitivities from the Monte Carlo runs and the blue solid line the median. The black solid line in figure (b) represents the XENON1T limit at 90\% C.L. [31] and the dark grey box highlights roughly the best-fit parameter space from Ref. [32]. 


\section{Conclusion}

The all-sky eROSITA survey allows us to search for decaying sterile neutrino and axionlikeparticle (ALP) signal from the Galactic halo. We generate mock data sets with background only, as well as expected sky maps with counts from decaying sterile neutrinos in the Galactic halo, with four years of observations. Following a likelihood analysis, we obtain stringent bounds on the mixing angle of sterile neutrinos $(\theta)$, as well as on the ALP-photon coupling $\left(g_{a \gamma \gamma}\right)$ and ALP-electron coupling $\left(g_{a e}\right)$.

In the case of sterile neutrinos, we will be able to probe a value for the mixing angle up to nearly two orders of magnitude below the best-fit value of the unidentified $3.5 \mathrm{keV}$ line [1] and one order of magnitude below the existing upper limits claimed in the literature [8, 23-29].

Moreover, we will be able to probe the ALP couplings to photons and electrons with values that are not yet excluded by X-ray observations, to the same degree of improvement as in the case of sterile neutrinos. We investigate both a general model for the ALP-to-photon coupling and an anomaly-free symmetry model for the ALP-to-electron coupling that has been proposed to explain the XENON1T excess of electron recoil events [31]. The XENON1T excess could possibly be explained by an ALP origin for an excess at $m_{a} \sim 3 \mathrm{keV}$, which might be well tested with eROSITA.

\section{References}

[1] E. Bulbul, M. Markevitch, A. Foster, R.K. Smith, M. Loewenstein and S.W. Randall, Detection of an unidentified emission line in the stacked $x$-ray spectrum of galaxy clusters, The Astrophysical Journal 789 (2014) 13.

[2] A. Boyarsky, M. Drewes, T. Lasserre, S. Mertens and O. Ruchayskiy, Sterile neutrino Dark Matter, Prog. Part. Nucl. Phys. 104 (2019) 1 [1807. 07938].

[3] A. Boyarsky, O. Ruchayskiy, D. Iakubovskyi and J. Franse, Unidentified line in x-ray spectra of the andromeda galaxy and perseus galaxy cluster, Physical Review Letters 113 (2014) .

[4] A. Boyarsky, J. Franse, D. Iakubovskyi and O. Ruchayskiy, Checking the dark matter origin of a 3.53 kev line with the milky way center, Phys. Rev. Lett. 115 (2015) 161301.

[5] A. Neronov, D. Malyshev and D. Eckert, Decaying dark matter search with nustar deep sky observations, Physical Review D 94 (2016) .

[6] O. Urban, N. Werner, S.W. Allen, A. Simionescu, J.S. Kaastra and L.E. Strigari, A suzaku search for dark matter emission lines in the x-ray brightest galaxy clusters, 2014.

[7] D. Malyshev, A. Neronov and D. Eckert, Constraints on 3.55 kev line emission from stacked observations of dwarf spheroidal galaxies, Phys. Rev. D 90 (2014) 103506.

[8] J.W. Foster, M. Kongsore, C. Dessert, Y. Park, N.L. Rodd, K. Cranmer et al., A deep search for decaying dark matter with xmm-newton blank-sky observations, 2021.

[9] C. Dessert, N.L. Rodd and B.R. Safdi, The dark matter interpretation of the 3.5-kev line is inconsistent with blank-sky observations, Science 367 (2020) 1465-1467. 
[10] I.G. Irastorza and J. Redondo, New experimental approaches in the search for axion-like particles, Prog. Part. Nucl. Phys. 102 (2018) 89 [1801.08127].

[11] J.F. Navarro, C.S. Frenk and S.D.M. White, A Universal density profile from hierarchical clustering, Astrophys. J. 490 (1997) 493 [astro-ph/9611107].

[12] J.I. Read, O. Agertz and M.L.M. Collins, Dark mat-

ter cores all the way down, Monthly Notices of the Royal Astronomical Society 459 (2016) 2573 [https://academic . oup. com/mnras/article-pdf/459/3/2573/8105757/stw713.pdf].

[13] D. Sicilian, N. Cappelluti, E. Bulbul, F. Civano, M. Moscetti and C.S. Reynolds, Probing the Milky Way's Dark Matter Halo for the $3.5 \mathrm{keV}$ Line, 2008.02283.

[14] K.N. Abazajian, Neutrinos in astrophysics and cosmology: Theoretical advanced study institute (tasi) 2020 lectures, 2021.

[15] T. Higaki, K.S. Jeong and F. Takahashi, The $7 \mathrm{keV}$ axion dark matter and the X-ray line signal, Phys. Lett. B 733 (2014) 25 [1402.6965].

[16] K. Nakayama, F. Takahashi and T.T. Yanagida, Anomaly-free flavor models for nambu-goldstone bosons and the x-ray line signal, Physics Letters B 734 (2014) 178-182.

[17] D. Lumb, R. Warwick, M. Page and A. De Luca, X-ray background measurements with xmm-newton epic, Astron. Astrophys. 389 (2002) 93 [astro-ph/0204147].

[18] P. Predehl, R. Andritschke, V. Arefiev, V. Babyshkin, O. Batanov, W. Becker et al., The erosita x-ray telescope on srg, Astronomy \& Astrophysics 647 (2021) A1.

[19] P. Predehl, R.A. Sunyaev, W. Becker, H. Brunner, R. Burenin, A. Bykov et al., Detection of large-scale $x$-ray bubbles in the milky way halo, Nature 588 (2020) 227-231.

[20] A. Merloni, P. Predehl, W. Becker, H. Böhringer, T. Boller, H. Brunner et al., erosita science book: Mapping the structure of the energetic universe, 2012.

[21] P.D. Serpico and G.G. Raffelt, Lepton asymmetry and primordial nucleosynthesis in the era of precision cosmology, Physical Review D 71 (2005) .

[22] J.F. Cherry and S. Horiuchi, Closing in on resonantly produced sterile neutrino dark matter, Physical Review D 95 (2017) .

[23] S. Horiuchi, P.J. Humphrey, J. Onorbe, K.N. Abazajian, M. Kaplinghat and

S. Garrison-Kimmel, Sterile neutrino dark matter bounds from galaxies of the Local Group, Phys. Rev. D 89 (2014) 025017 [1311.0282].

[24] K.C.Y. Ng, S. Horiuchi, J.M. Gaskins, M. Smith and R. Preece, Improved Limits on Sterile Neutrino Dark Matter using Full-Sky Fermi Gamma-Ray Burst Monitor Data, Phys. Rev. D 92 (2015) 043503 [1504 . 04027]. 
[25] K. Perez, K.C.Y. Ng, J.F. Beacom, C. Hersh, S. Horiuchi and R. Krivonos, Almost closing the vMSM sterile neutrino dark matter window with NuSTAR, Phys. Rev. D 95 (2017) 123002 [1609.00667].

[26] K.C.Y. Ng, B.M. Roach, K. Perez, J.F. Beacom, S. Horiuchi, R. Krivonos et al., New Constraints on Sterile Neutrino Dark Matter from NuST AR M31 Observations, Phys. Rev. D 99 (2019) 083005 [1901.01262].

[27] K.N. Abazajian, Sterile neutrinos in cosmology, Physics Reports 711-712 (2017) 1-28.

[28] A. Caputo, M. Regis and M. Taoso, Searching for Sterile Neutrino with X-ray Intensity Mapping, JCAP 03 (2020) 001 [1911.09120].

[29] B.M. Roach, K.C. Ng, K. Perez, J.F. Beacom, S. Horiuchi, R. Krivonos et al., Nustar tests of sterile-neutrino dark matter: New galactic bulge observations and combined impact, Physical Review D 101 (2020) .

[30] A. Neronov and D. Malyshev, Toward a full test of themsmsterile neutrino dark matter model with athena, Physical Review D 93 (2016) .

[31] E. Aprile, J. Aalbers, F. Agostini, M. Alfonsi, L. Althueser, F. Amaro et al., Excess electronic recoil events in xenon1t, Physical Review D 102 (2020).

[32] F. Takahashi, M. Yamada and W. Yin, XENON1T Excess from Anomaly-Free Axionlike Dark Matter and Its Implications for Stellar Cooling Anomaly, Phys. Rev. Lett. 125 (2020) 161801 [2006.10035].

[33] M.M. Miller Bertolami, B.E. Melendez, L.G. Althaus and J. Isern, Revisiting the axion bounds from the Galactic white dwarf luminosity function, JCAP 10 (2014) 069 [1406 . 7712].

[34] N. Viaux, M. Catelan, P.B. Stetson, G.G. Raffelt, J. Redondo, A.A.R. Valcarce et al., Neutrino and axion bounds from the globular cluster $m 5$ (ngc 5904), Physical Review Letters 111 (2013) . 